\title{
COVID-19 e miocardite: uma possível consequência cardíaca após a infecção pelo SARS-CoV-2
}

COVID-19 and myocarditis: on a possible consequence after SARS-CoV-2 infection

COVID-19 y miocarditis: una posible consecuencia cardíaca después de la infección por SARS-CoV-2

Izabella Guedes Ferreira ${ }^{1 *}$, Ana Carolina Tassara Azevedo ${ }^{1}$, Ana Flávia Amaral Durigueto ${ }^{1}$, Isabella Maise de Andrade Rodrigues Sóter ${ }^{1}$, Juliana de Oliveira Miranda Simões ${ }^{1}$, Laura Lustosa Soares ${ }^{1}$, Thiago Santiago Ferreira ${ }^{1}$.

\section{RESUMO}

Objetivo: Revisar sobre a relação entre a COVID-19 e a miocardite, levando em consideração os mecanismos fisiopatológicos, métodos diagnósticos e abordagem terapêutica. Revisão bibliográfica: O SARS-CoV-2 pode invadir o organismo do hospedeiro e desenvolver um quadro composto por três fases: a fase inicial da infecção, a pulmonar e a de super inflamação. É nessa última, que geralmente ocorrem os danos ao sistema cardiovascular, em que o vírus se prolifera no sistema imunológico e invade os cardiomiócitos, ocasionando uma inflamação miocárdica e remodelação cardíaca e, consequentemente, a cardiomiopatia. Exames complementares como ecocardiografia transtorácica, eletrocardiograma, dosagem da troponina $\mathrm{T}$ ultrassensível e a ressonância magnética cardíaca são importantes na investigação diagnóstica da miocardite. Quanto ao tratamento, deve-se levar em consideração o quadro clínico do paciente e envolve terapias de suporte e objetiva alívio sintomático. Considerações finais: A miocardite é uma complicação cardiológica da infecção pelo SARS-CoV-2 cada vez mais relatada, sendo fundamental, por parte do profissional de saúde, o conhecimento da fisiopatologia e quadro clínico característico da doença, bem como os fatores de risco relacionados, para diagnóstico e intervenções precoces.

Palavras chaves: SARS-CoV-2, COVID-19, Miocardite.

\begin{abstract}
Objective: Review the correlation between COVID-19 and myocarditis, taking into account the pathophysiological mechanisms, diagnostic methods and therapeutic approach. Bibliographic review: SARS-CoV-2 can invade the host's organism and develop a condition composed of three phases: the initial phase of infection, pulmonary and super inflammation. It is in the latter that damage to the cardiovascular system usually occurs, in which the virus proliferates in the immune system and invades the cardiomyocytes, leading to a myocardial inflammation and cardiac reshuffle and consequently myocarditis. Complementary exams like transthoracic echocardiography, electrocardiogram, ultrasensitive T Troponin and cardiological magnetic resonance are important in the diagnostic investigation of myocarditis. As to the treatment, the clinical situation should be considered and involves support therapies with relief symptoms as a goal. Final considerations: Myocarditis is an increasingly reported cardiology complication of SARS-CoV-2 infection, and it is essential that healthcare professionals have knowledge of the pathophysiology and clinical features of the disease, as well as the related risk factors, for early diagnosis and interventions.
\end{abstract}

Keywords: SARS-CoV-2, COVID-19, Myocarditis.

${ }^{1}$ Pontifícia Universidade Católica de Minas Gerais (PUC Minas), Betim - MG.

*E-mail: izabellaguedes@outlook.com

SUBMETIDO EM: 7/2021

ACEITO EM: 7/2021

PUBLICADO EM: 9/2021 


\section{RESUMEN}

Objetivo: Abordar la correlación entre COVID-19 y la miocarditis, a consideración de los mecanismos fisiopatológicos, los métodos diagnósticos y el abordaje terapéutico. Revisión bibliográfica: EI SARS-CoV2 puede invadir el organismo del huésped y desarrollar un cuadro compuesto por tres fases: la inicial, el pulmón y la super inflamación. Es en este último donde suele producirse daño al sistema cardiovascular, en el que el virus prolifera en el sistema inmunológico e invade los cardiomiocitos, provocando inflamación y remodelado cardíaco $y$, en consecuencia, miocardiopatía. Las pruebas complementarias como la ecocardiografía transtorácica, el electrocardiograma, la medición de la troponina $T$ ultrasensible y la resonancia magnética cardíaca son importantes en la investigación diagnóstica. En cuanto al tratamiento, hay que tener en cuenta la situación clínica del paciente e implica terapias de apoyo y alivio sintomático objetivo. Consideraciones finales: La miocarditis es una complicación cada vez más notificada de la infección por SARS-CoV-2, y es esencial que el profesional conozca la fisiopatología y las características clínicas, así como los factores de riesgo involucrados, para un diagnóstico temprano e intervenciones.

Palabras clave: SARS-CoV-2, COVID-19, Miocarditis.

\section{INTRODUÇÃO}

A COVID-19, causada pelo patógeno SARS-CoV-2, teve seu primeiro caso reportado na cidade de Wuhan, em 2019, na China, e em março de 2020 foi classificada como pandemia pela Organização Mundial de Saúde (WHO, 2020). Existe um grande espectro de possíveis manifestações clínicas causadas por essa doença, variando entre pacientes assintomáticos, outros com acometimento respiratório e casos mais graves, com comprometimento de outros sistemas, como o cardiovascular. Entre as afecções cardiovasculares relacionadas à infecção pelo SARS-CoV-2 já relatadas na literatura, a miocardite se apresenta como uma patologia importante devido à frequência e suas possíveis complicações. Estima-se que 7 a $23 \%$ dos casos relatados em Wuhan, evoluíram com esse tipo de complicação (PIRZADA A, et.al., 2020).

De acordo com Shah $\mathrm{N}$ et al. (2018), a miocardite é uma inflamação do musculo cardíaco que pode resultar em disfunção cardíaca e arritmias. Trata-se se uma condição não isquêmica na qual existe presença de infiltrado inflamatório no miocárdio, levando a uma agressão e lesão dos cardiomiócitos. Ela pode ocorrer em um contexto isolado ou associada a doença sistêmica, geralmente de origem infecciosa ou imunológica. Segundo Yokoo P, et al. (2020), devemos suspeitar que o paciente apresenta essa condição toda vez que ele apresentar febre acompanhada de insuficiência cardíaca ou arritmias de início súbito.

Segundo Canter CE e Simpson KE (2014), para fazer o diagnóstico de miocardite é necessário, além dos sinais clínicos sugestivos da doença, a caracterização imunológica e histológica dos cardiomiócitos através da biópsia endomiocárdica. Entretanto, na realidade, pouquíssimas pessoas sob suspeita de inflamação do cardiomiócitos, tem de fato, o quadro confirmado através de biópsia, sendo, portanto, esse diagnóstico realizado embasado somente em hipóteses e indícios.

No contexto em que são realizadas as biópsias, mesmo sob inúmeras ressalvas devido a fraca correlação clínica, o Critério de Dallas, criado em 1980, é uma das ferramentas utilizadas para o diagnóstico de miocardite. Nesse critério, é tido como condições necessárias para a confirmação do quadro, a presença de infiltrado inflamatório no miocárdio com necrose e/ou degeneração dos miócitos adjacentes não decorrentes de danos isquêmicos associados à doença arterial coronariana. Ademais, é ainda sugerido que o diagnóstico de miocardite seja estendido a todo e qualquer estado pró inflamatório, ou seja, ao primeiro sinal de uma maior expressão de citocinas e antígenos de histocompatibilide (MONTERA MW, et. al., 2013).

A miocardite pode ser causada por diversos patógenos e a característica do infiltrado inflamatório presente na lesão é fundamental para sua classificação, sugerindo sua etiologia. Dito isso, as miocardites podem ser classificadas como neutrofílicas, eosinofílicas, linfocitocitárias (mononucleares), granulomatosas ou de células gigantes (MONTERA MW, et. al.,2013).

Dentre as causas de miocardite, a mais comum do nosso meio é a viral. A presença de uma infecção por vírus, assim como nas reações imunológicas, induz a produção de um infiltrado inflamatório 
predominantemente mononuclear nos cardiomiócitos. Caso a invasão do hospedeiro seja por bactéria ou por uma reação de hipersensibilidade, observa-se a presença de infiltrado polimorfonuclear. Infecções por helmintos causam, em sua maioria, infiltrado granulomatoso, podendo observar fragmentos do parasita. Sarcoidose também se apresenta com infiltrado inflamatório granulomatoso. Por fim, a miocardite de Fiedler apresenta infiltrado com células gigantes (ASKIN L, et al., 2020; MONTERA MW, et. al.,2013).

Inicialmente acreditava-se que o vírus Coxsackie era o maior responsável por casos de miocardites virais. Posteriormente, os enterovirus e adenovírus foram encontrados em biopsias de pacientes com suspeita de miocardite e atualmente são considerados os vírus mais prevalentes nestes casos. Outros vírus como parvovírus-B19, herpes simples, vírus da hepatite C (HCV), citomegalovírus (CMV), Epstein-Barr (EBV), pólio, influenza, HIV, Caxumba, varicela, sarampo e, finalmente, SARS-CoV-2 podem infectar as células do coração. Normalmente, suspeita-se de MV sempre que há descompensação cardíaca ou arritmias, dias ou semanas após uma infecção viral (MONTERA MW, et. al.,2013).

A MV pós infecção por SARS-CoV-2, no contexto atual da pandemia, vem sendo cada vez mais discutida, uma vez que essa condição está sendo frequentemente relatada, principalmente em doentes graves. Um aspecto significativo que tem sido descrito, refere-se ao fato de que pacientes com quadros sugestivos de injúria miocárdica estão associados à maior necessidade de suporte ventilatório e mortalidade intra-hospitalar, por isso, a relevância do tema no contexto de saúde pública. Alguns métodos de imagem podem ser utilizados para elucidar o diagnóstico, tais como Tomografia Computadorizada de Tórax, Ecocardiograma e Ressonância Magnética (YOKOO P, et al., 2020).

Assim, pode-se afirmar que a miocardite relacionada ao COVID-19 é um tema de extrema relevância atualmente, tendo em vista a gravidade das possíveis complicações da doença. Desta forma, esse trabalho tem como objetivo explanar sobre a COVID-19, dando enfoque na miocardite, como uma importante complicação cardiovascular, abordando sua fisiopatologia, repercussões clínicas, diagnóstico e tratamento, bem como os fatores de risco relacionados com a gravidade dos quadros. Por fim, este artigo consiste em uma revisão narrativa, realizada por meio de artigos publicados na base de dados da PUBMED.

\section{REVISÃO BIBLIOGRÁFICA}

\section{Relatos de casos de associação entre COVID-19 e miocardite}

Em um estudo realizado por Zeng $\mathrm{JH}$, et al. (2020), foi reportado o primeiro caso sobre miocardite associado ao quadro de COVID-19. Nesse estudo foi apresentado o caso clínico de um paciente de 63 anos, tabagista, sem outras comorbidades prévias, com histórico de viagem recente à China e que apresentava, no momento de sua internação, expectoração branca pegajosa, febre de $39,3{ }^{\circ} \mathrm{C}$, falta de ar e sensação de aperto no peito após esforço físico e saturação de $91,8 \%$. Foi confirmada laboratorialmente a infecção pelo novo coronavírus, envolvendo acometimento renal e hepático.

Exames cardíacos revelaram ainda, segundo o estudo de Zeng JH, et al. (2020), elevação de troponina I, mioglobina e peptídeo natriurético cerebral n-terminal, além do eletrocardiograma (ECG) com ritmo em taquicardia sinusal sem supradesnivelamento do segmento ST. À ecocardiografia, foi visto que o ventrículo esquerdo estava aumentado somado à uma baixa fração de ejeção, devido a uma discinesia do músculo cardíaco. Assim, o paciente foi diagnosticado com um quadro de miocardite fulminante e síndrome de disfunção de múltiplos órgãos, devido ao quadro inicial de pneumonia grave. Apesar de intervenções com antivirais, corticóides, antibióticos e imunomoduladores e outras medidas de suporte, inclusive oxigenação por membrana extracorpórea e drogas vasoativas, o paciente evoluiu com choque séptico após trinta e três dias de internação.

Desde então, novos casos correlacionando a COVID-19 à miocardite foram descritos na literatura. Diferentemente do primeiro, nem todos tiveram desfechos desfavoráveis, como indica o caso conduzido por Inciardi RM, et al. (2020). De acordo com os autores, uma mulher de 53 anos, previamente hígida apresentou fadiga associada à tosse seca e febre e hipotensão. Foi confirmada infecção pelo SARS-Cov-2 e o ECG mostrou elevação difusa do segmento ST, além de apresentar elevação de troponina e BNP. Obstrução 
coronariana foi descartada pela angiografia e a ressonância magnética cardíaca indicou aumento de espessura da parede, com hipocinesia biventricular difusa e disfunção ventricular esquerda grave, além de efusão pericárdica circunferencial, sendo os achados condizentes com miopericardite aguda.

Diante dos achados, segundo o estudo de Inciardi RM, et al. (2020), a paciente chegou a precisar de inotrópicos devido à hipotensão, além do uso de antivirais, esteróides, cloroquina e medicamentos para insuficiência cardíaca. Houve progressiva melhora clínica, inclusive com melhora da fração de ejeção quando submetida à ecocardiografia no sexto dia de internação.

Os estudos publicados até então também mostram uma variedade tanto demográfica quanto sobre a apresentação clínica. Por exemplo, Kim IC, et al. (2020) relataram o caso de uma jovem de 21 anos que apresentava importante quadro gripal, com confirmação de infecção pelo SARS-Cov-2. Com resultados alterados de troponina e BNP, foram realizados exames de imagem que evidenciaram achados compatíveis com miocardite. Outro estudo conduzido por Gnecchi M, et al. (2020) trouxe o caso de um jovem de 16 anos que não apresentava nenhum outro sintoma gripal além de febre e também intensa dor torácica. Foi confirmado COVID-19 e exames cardíacos de imagem condiziam com o diagnóstico de miocardiopatia aguda. O paciente apresentou boa evolução clínica e recebeu alta após 12 dias de internação.

Por fim, o artigo de Zeng JH, et al. (2020) traz ainda que em estudos de autópsia em pacientes infectados pelo coronavírus mostram uma infiltração mononuclear no tecido cardíaco e a rápida recuperação da função cardíaca presenciada em alguns pacientes, pode estar relacionada a uma possível tempestade de citocinas, as quais podem ocasionar aumento da parede vascular pela crescente permeabilidade das células. Somado a isso, a severa hipóxia experimentada por muitos pacientes acometidos pela COVID-19 pode também acrescer a resposta inflamatória e gerar danos ao coração.

Um estudo realizado por Sy Ho J, et al. (2020), analisou 31 relatos de casos publicados envolvendo 51 pacientes com suspeita de miocardite induzida pelo SARS-CoV-2, e encontraram dados semelhantes ao caso clínico relatado por Zeng $\mathrm{JH}$, et al. (2020). A maioria dos pacientes que evoluíram com miocardite apresentaram febre, dispneia, dor no peito, elevação sérica de marcadores cardíacos e inflamatórios, além de alterações na ecocardiografia como disfunção ventricular esquerda e hipocinesia.

\section{Fisiopatologia da infecção pela COVID-19}

No final do segundo semestre de 2019, na cidade de Wuhan, na China, novos casos de pneumonia começaram a aparecer, oriundos de uma cepa de coronavírus jamais identificada em humanos. Ainda naquele ano as autoridades chinesas alertaram a Organização Mundial de Saúde sobre essa nova variante viral e desde então, incansáveis estudos têm sido feitos em busca de entender os potenciais danos que esse vírus pode causar à população. Em janeiro do ano seguinte, a OMS declarou que o novo coronavírus constituía uma emergência de saúde pública de importância internacional e em 11 de março de 2020 a COVID-19 foi decretada como uma pandemia (ORGANIZAÇÃO PAN-AMERICANA DA SAÚDE (OPAS), 2020).

As doenças causadas pelas diversas famílias de coronavírus podem gerar uma variedade de sintomas, desde os mais leves aos mais graves e não diferente, acontece com o SARS-CoV-2. As pessoas que contraem esse vírus podem apresentar diversos sintomas tais como dor de garganta, congestão nasal, rinorreia, tosse seca persistente, hiposmia ou mesmo anosmia, ageusia, diarreia, febre, cansaço, e nos casos mais graves, podem surgir sintomas como dispneia, dor torácica e perda dos movimentos (COLOMBO CSSS, et al., 2020).

A instalação do quadro é dividida em três tempos: fase inicial da infecção, quando o vírus invade o organismo e começa a se replicar, a fase pulmonar, na qual tem-se o comprometimento do sistema respiratório e, por último, a fase de hiper-inflamação, na qual ocorrem danos a órgãos secundários. Diante disso, na terceira fase da doença, o sistema cardiovascular pode ser afetado direta ou indiretamente de forma grave, levando a lesões do miocárdio, arritmias, miocardite, insuficiência cardíaca congestiva, cardiomiopatia por estresse, síndrome coronariana aguda, a qual pode estar relacionada ao estado pró trombótico gerado pela doença e, até mesmo, choque cardiogênico. Nesse contexto, a lesão cardíaca se tornou uma característica proeminente do COVID-19, acometendo uma parcela significativa dos infectados e contribuindo, com grande relevância, para o óbito desses pacientes (COLOMBO CSSS, et al., 2020). 
Em relação à fisiopatologia da infecção, a proteína spike da superfície do SARS-CoV-2 se liga ao receptor da Enzima de Conversão da Angiotensina II (ECA-2) que está por todo o corpo, em especial nas células epiteliais da traqueia e brônquios, nos pneumócitos tipo II, macrófagos, células epiteliais do intestino, nas células renais, no endotélio vascular e nas células musculares, no endotélio das artérias coronárias, nos cardiomiócitos, fibroblastos e adipócitos epicárdicos, promovendo um aumento dos níveis de angiotensina II no Sistema Renina Angiotensina Aldosterona (SRAA), devido à ausência de feedback negativo da angiotensina (1-7) (SOUZA DSM, et al., 2021).

Dessa forma, diante da falta de conversão de angiotensina I e II em angiotensina (1-7), ocorre um déficit na atividade vasodilatadora, bem como na atividade anti-inflamatória, antifibrótica e ainda, uma falha na redução da pressão arterial. Além disso, o excesso de angiotensina II, por meio de suas ações, como promover vasoconstrição, reabsorver sódio renal e excretar potássio, sintetizar aldosterona e elevar a pressão arterial, pode causar diversas reações adversas, com destaque para disfunção e hipertrofia do miocárdio, fibrose intersticial, inflamação aumentada, estresse oxidativo e distúrbios da coagulação sanguínea (SOUZA DSM, et al., 2021).

Ademais, quando a angiotensina II se liga ao receptor AT1, ocorre uma promoção do estado próinflamatório, pró oxidativo, pró apoptóico, pró fibrótico, vasoconstritor e promove ainda, um aumento da permeabilidade vascular, favorecendo a instalação de edema pulmonar. Além dessa hiperestimulação dos receptores AT1 devido à alta disponibilidade de angiotensina II, essa última é ainda convertida em angiotensina IV, a qual promove, por meio do receptor AT4, estado trombótico, o qual se correlaciona com a instalação de quadros respiratórios e cardíacos graves (SOUZA DSM, et al., 2021).

Figura 1- SARS-CoV- 2 e sua ação na cascata do Sistema Renina Angiotensina Aldosterona.

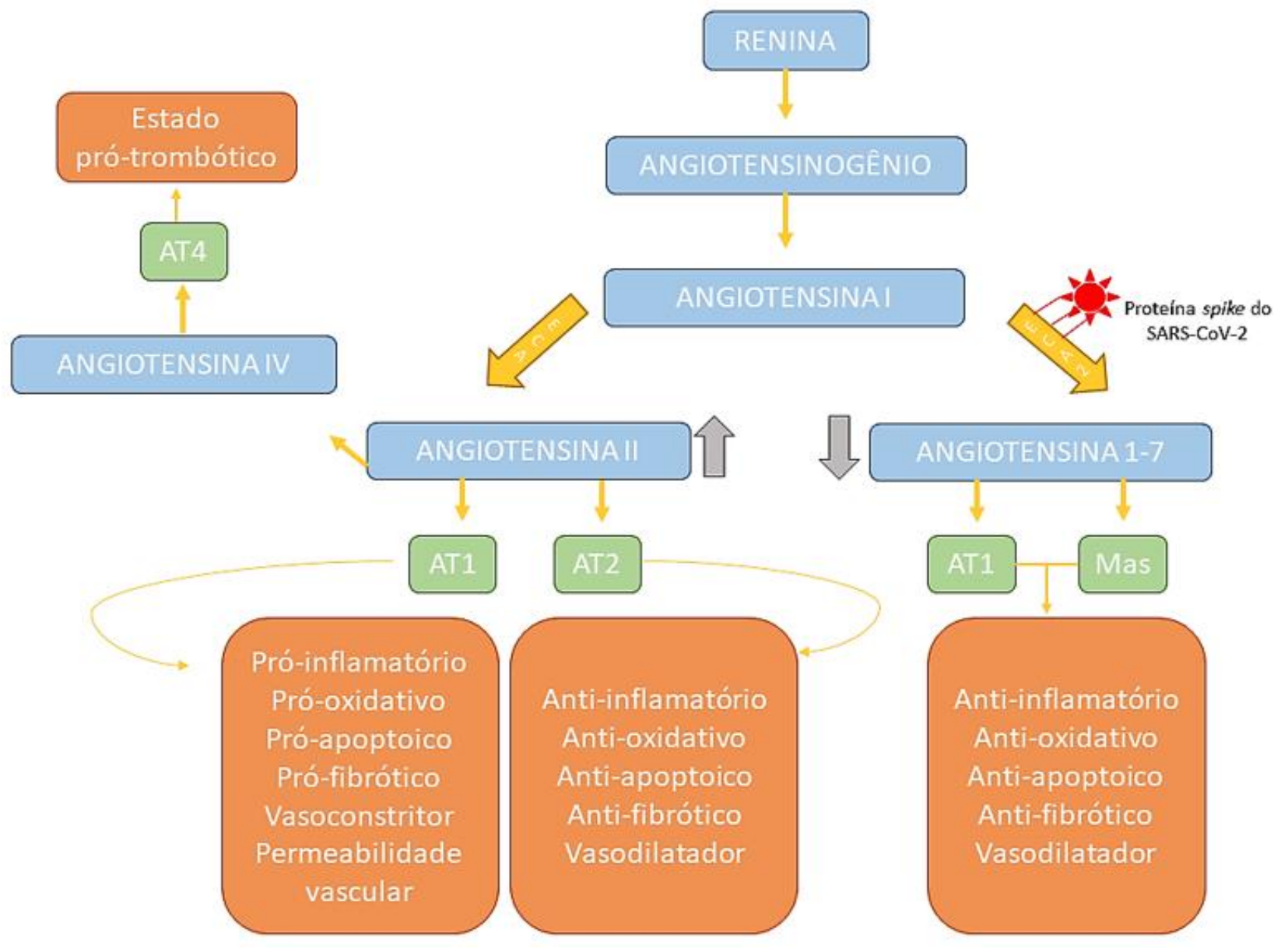

Fonte: Ferreira IG, et al., 2021; dados baseados na: Revista Brasileira de Hipertensão, 2021. Imagem criada pelos autores pelo Microsoft Power Point ${ }^{\circledR}$. 
Diante disso, a fisiopatologia do envolvimento cardíaco com a COVID- 19 é resultado da interação entre o vírus (SARS-CoV-2) com o sistema imunológico do hospedeiro. O vírus, pode se proliferar no sistema imunológico do hospedeiro e invadir os cardiomiócitos através de receptores específicos de replicação viral, que se persistirem, podem levar a inflamação miocárdica e gerar a remodelação cardíaca, levando ao desenvolvimento de cardiomiopatia (VELASCO IT, et al., 2019).

\section{Miocardite como consequência da infecção pelo SARS-CoV-2}

As complicações relacionadas ao sistema cardíaco são mais frequentes em indivíduos com um histórico prévio de comorbidades, como diabetes, hipertensão, aumento da massa corporal e presença de idade avançada (COSTA IBSS, et al.,2020). Os mecanismos são multifatoriais, e envolvem um desbalanço entre a demanda e reserva metabólica. A associação entre a lesão miocárdica e a infecção pelo SARS-Cov-2 vem sendo relatada na literatura, mas ainda são necessários mais estudos que demonstrem como a fisiopatologia da lesão miocárdica interfere no prognóstico dos pacientes com a SARS-Cov-2 (NASCIMENTO JHP, et al., 2021).

Os sinais e sintomas da miocardite em pacientes com COVID-19 são amplos, desde apresentações leves com fadiga, febre e mialgia até situações de emergência como arritmias ventriculares ou sinais de choque cardiogênico. Em um contexto de pandemia, o profissional de saúde deve estar atento a piora abrupta dos sintomas respiratórios como dispneia, dor torácica e sinais de insuficiência cardíaca bem como, o aumento inexplicado de marcadores de necrose miocárdica. Foram descritos na literatura, casos de miocardite fulminante, com forte acometimento ventricular e rápida evolução. Frequentemente, nesses pacientes, foram encontrados elevação de troponina, proteína $C$ reativa e alterações no eletrocardiograma (ECG). Em relação ao BNP/NT-probNP, alguns estudos também sugerem ser um marcador prognóstico importante. Tais alterações predispõem ao paciente a Síndrome de angústia respiratória do adulto- SDRA e aumentam a mortalidade (COSTA IBSS, et al., 2020).

Neste contexto, a solicitação de exames complementares, como a ecocardiografia transtorácica, faz-se necessária. A ecocardiografia, geralmente, é a primeira técnica de imagem realizada à beira do leito do paciente. Essa técnica é boa, pois pode ser utilizada tanto em pacientes estáveis quanto instáveis. Entretanto, apresenta algumas limitações no diagnóstico da miocardite, pois as disfunções sistólicas ventriculares globais e regionais não são marcadores exclusivos da miocardite aguda, podendo ser encontradas em outras patologias. Além disso, os pacientes com miocardite podem apresentar fração de ejeção ventricular esquerda normal, o que, não exclui a doença. Em casos fulminantes, é comum o aparecimento do espessamento da parede. Outro aspecto que deve ser considerado, é o contexto prévio do paciente, tendo em vista a possibilidade de uma disfunção ventricular pré-existente que deve ser sempre levada em consideração, especialmente se o paciente tiver fatores de risco cardiovasculares conhecidos (MELE D, et al., 2021).

Além disso, é importante que o profissional de saúde tenha em mãos o ECG. São achados frequentes em pacientes com miocardite: alterações do segmento ST (geralmente a depressão), inversão da onda T, distúrbios da condução e arritmias. Outro exame fundamental a ser solicitado é a Troponina T Ultrassensível (TTU), que vem sendo usada, inclusive, como um valor prognóstico, visto que ela é um importante marcador de lesão miocárdica. Nesse contexto, a dosagem de TTU na fase subaguda do quadro clínico é de grande valor prognóstico, servindo como uma ferramenta de estratificação de risco e de orientação quanto a indicação de Ressonância Magnética Cardíaca (RMC), a fim de uma investigação mais coesa do acometimento cardíaco do paciente em questão (COLOMBO CSSS, et al.,2020).

A RMC é um teste sensível e não invasivo para confirmação diagnóstica (YOKOO P, et al.,2020). Tratase de um excelente exame para identificação de edema, inflamação, fibrose miocárdica além de possibilitar a diferenciação entre miocardite de etiologia isquêmica ou não isquêmica. De acordo com Radunski UK, et al. (2014), a RMC é a técnica de imagem de escolha para pacientes com suspeita de miocardite. Entretanto, é de alto custo, baixa disponibilidade e deve ser contraindicado em pacientes com quadros de insuficiência cardíaca grave, choque circulatório, bloqueio átrio ventricular de alto grau e arritmias ventriculares (COLOMBO CSSS, et al., 2020; PIRZADA A, et.al., 2020). 
Importante enfatizar que biópsia endomiocárdica é considerada o exame "padrão ouro" para pacientes com miocardite. As alterações histopatológicas no tecido miocárdico mostraram tipicamente um infiltrado inflamatório do miocárdio com presença de linfócitos, neutrófilos, eosinófilos, células gigantes, granulomas ou uma mistura. A inflamação do miocárdio pode ser difusa ou focal. A inflamação pode se estender para o pericárdio, causando pericardite (COLOMBO CSSS, et al., 2020).

Um aspecto relatado na literatura é que devido à alta taxa de transmissão interpessoal do SARS-CoV-2 no ambiente, está ocorrendo um alto controle hospitalar a fim de evitar a contaminação de funcionários e de garantir a segurança dos pacientes. Com isso, alguns exames complementares vêm sendo adiados, comprometendo, dessa forma, a capacidade de determinar os mecanismos de lesão miocárdica (NASCIMENTO JHP, et al., 2021).

Segundo Krasic S, et al. (2021), 50\% das miocardites têm resolução espontânea. Quando se faz necessário, o tratamento da miocardite deve ser baseado na terapia para disfunção ventricular e estado clínico do paciente, sendo o cuidado direcionado para o suporte e o alívio sintomático (MELE D, et al.,2021). As indicações de um desfibrilador cardioversor implantável são semelhantes às da cardiomiopatia não isquêmica; no entanto, um desfibrilador cardioversor implantável deve ser mantido na fase aguda da miocardite para permitir a recuperação da fração de ejeção do ventrículo esquerdo (ALI-AHMED F, et al., 2020).

Apesar do assunto ser bastante divergente na literatura, alguns autores sugerem o uso de corticosteroides em altas doses e imunoglobulinas intravenosas (IGIV), devido à concepção de que em estágios graves da COVID-19 há uma difusa ativação imunológica e que o uso de corticoides seria uma boa opção para reduzir a atividade imunológica. As IGIV foram escolhidas para o tratamento, pois possuem uma ação multifatorial, e demonstraram não apenas efeitos antivirais, mas também efeitos anti-inflamatórios por suprimir citocinas inflamatórias. Entretanto, existem controvérsias na literatura, sendo, portanto, necessário mais estudos para o implemento na prática diária (MELE D, et al.,2021).

Era esperado que os agentes antivirais fossem eficazes em pacientes com COVID-19 como o remdesivir, ritonavir, lopinavir e até mesmo a hidroxicloroquina. Infelizmente, esses medicamentos tiveram pouco ou nenhum efeito na mortalidade geral. Portanto, faltam dados sobre a eficácia dos agentes antivirais no contexto da miocardite por COVID-19 (MELE D, et al.,2021).

Antes da pandemia da COVID-19, o transplante cardíaco e o suporte ventilatório mecânico eram uma opção de tratamento para pacientes com MV em estágio terminal. $O$ transplante, porém, não deve ser realizado em pacientes infectados pelo SARS-CoV-2 devido a infecção ativa e contínua provocada pelo vírus. Já o suporte ventilatório mecânico pode ser considerado uma boa opção para casos de miocardite fulminante (PIRZADA A, et al., 2020).

\section{CONSIDERAÇÕES FINAIS}

A COVID-19 tem sido associada à miocardite, aumentando a gravidade e a mortalidade da doença. A detecção de injúria miocárdica deve acontecer de maneira precoce com o objetivo de evitar complicações e óbitos. Exames de imagem e laboratoriais são opções que auxiliam no diagnóstico, mas a biópsia endomiocardica é o exame padrão ouro para detectar a miocardite. O tratamento deve ser baseado na terapia da disfunção cardíaca apresentada pelo paciente. O uso de corticoesteróides em altas doses ainda é controverso na literatura. Os agentes antivirais não demonstraram impacto na mortalidade geral. Tendo em vista o exposto, fica clara a gravidade da miocardite associada a infecção pela COVID-19 e a importância de mais estudos que busquem a detecção e tratamento precoce desta complicação.

\section{REFERÊNCIAS}

1. ALI-AHMED F, et al. Sudden cardiac death in patients with myocarditis: evaluation, risk stratification, and management. American Heart Journal, 2020; 220: 29-40.

2. ASKIN L, et al. O Efeito da Doença de Coronavírus 2019 nas Doenças Cardiovasculares. Arquivos Brasileiros de Cardiologia, 2020; 114(5): 817-822. 
3. CANTER CE, SIMPSON KE. Diagnosis and Treatment of Myocarditis in Children in the Current Era. Circulation. 2014; 129: $115-128$.

4. COLOMBO CSSS, et al. Posicionamento sobre Avaliação Pré-participação Cardiológica após a Covid-19: orientações para retorno à prática de exercícios físicos e esportes. Arquivos Brasileiros de Cardiologia, 2020; 1-30.

5. COSTA IBSS, et al. O Coração e a COVID-19: o que o cardiologista precisa saber. Arquivos Brasileiros de Cardiologia, 2020; 114(5): 805-816.

6. GNECCHI M, et al. Myocarditis in a 16-year-old boy positive for SARS-CoV-2. The Lancet. 2020. 395 (10242): 116.

7. INCIARDI RM, et al. Cardiac Involvement in a Patient with Coronavirus Disease 2019 (COVID-19). JAMA Cardiology. $2020 ; 5(7): 1-6$.

8. KIM IC, et al. COVID-19- related myocarditis in a 21-year-ols female patient. OUP Public Health Emergency Collection. 2020; 41(19): 1859.

9. KRASIC S, et al. Could the unfortunate outcome of pediatric acute myocarditis be predicted? Factors contributing to a poor outcome in myocarditis. Revista portuguesa de cardiologia, 12 junho 2021.

10. MAHRHOLDT H, et al. Presentation, Patterns of Myocardial Damage, and Clinical Course of Viral Myocarditis. Circulation, 2006; 114: 1581-1590.

11. MELE D, et al. Myocarditis in COVID-19 patients: current problems. Internal And Emergency Medicine. Springer Science and Business Media LLC, 2021; 23: 1-8.

12. MONTERA MW, et al. I Diretriz Brasileira de miocardite e pericardite. Arquivo Brasileiro de Cardiologia, Brasil. 2013.

13. NASCIMENTO, JHP, et al. COVID-19 e Injúria Miocárdica em UTI Brasileira: alta incidência e maior risco de mortalidade intra-hospitalar. Arquivos Brasileiros de Cardiologia, 2021; 116(2): 275-282.

14. ORGANIZAÇÃO PAN-AMERICANA DA SAÚDE (OPAS). Folha Informativa sobre COVID-19. 2020.

15. PIRZADA A, et al. COVID-19 e Miocardite, o que sabemos até agora? CJC Open, 2020; 2(4): $278-285$.

16. RADUNSKI UK, et al. CMR in Patients With Severe Myocarditis: Diagnostic Value of Quantitative Tissue Markers Including Extracellular Volume Imaging. JACC: cardiovascular imaging, 2014; 7(7): 667-75.

17. SHAH N, et al. Myocarditis in the Athlete. American College of Cardiology, 2018.

18. SOUZA DSM, et al. Na COVID-19 é real a não suspensão dos inibidores do sistema renina angiotensina aldosterona? Revista Brasileira de Hipertensão, 2021; 28(1): 20-6.

19. SY HO J et al. Coronavirus-induced myocarditis: A meta-summary of cases. Hart e Lung 2020; 49: 681-685.

20. VELASCO IT, et al. Medicina de emergência: abordagem prática, 2019.

21. WORD HEALTH ORGANIZATION (WHO). Director-General's opening remarks at the media briefing on COVID. 2020. Disponível em: https://www.who.int/director-general/speeches/detail/who-director-general-s-opening-remarks-at-themedia-briefing-on-covid-19---23-october-2020. Acesso em: 10 jul. 2021.

22. YOKOO $P$, et al. COVID-19 myocarditis: a case report. einstein, 2020; 18.

23. ZENG JH, et al. First case of COVID-19 complicated with fulminant myocarditis: a case report and insights. Infection, 2020; 48: 773-777. 